\title{
The Minimal Kirchhoff Index Of Theta Shape Graph
}

\author{
Xiaomin Ren ${ }^{1}$, Jinyu Zou ${ }^{2}$ and Haizhen Ren ${ }^{1+}$ \\ ${ }^{1}$ Department of Mathematics, Qinghai Normal University, Xining 810008, Qinghai, China \\ ${ }^{2}$ Department of Basic Courses, Qinghai University, Xining 810016, Qinghai, China
}

\begin{abstract}
The resistance distance between any two vertices of a connected graph $\mathrm{G}$ is defined as the effective resistance between them in the electrical network constructed from $G$ by replacing each edge of $G$ with unit resistor. Kirchhoff index is a structure-descriptor based on resistance distance. For the theta shape graphs(a specified class of bicycle graphs), the ordering rations of their Kirchhoff index remain open. In this paper, some new ordering relations are obtained by three graph transformations, and the minimal Kirchhoff index and the corresponding graph in this class of graphs is also discussed.
\end{abstract}

Keywords: electrical network, resistance distance, Kirchhoff index, theta shape graph

\section{Introduction}

On the basis of electrical network theory, Klein and Randic[1] introduced the novel concept of resistance distance. Let $\mathrm{G}$ be a connected graph with vertices labelled as $1,2, \ldots, \mathrm{n}$. They view $\mathrm{G}$ as an electrical network $\mathrm{N}$ by considering each edge of $\mathrm{G}$ as a unit resistor, then the resistance distance between vertices $\mathrm{i}$ and $j$, denoted by $r_{i j}$, is defined to be the effective resistance between nodes $i$ and $j$ as computed with Ohm's law in N. The Kirchhoff index of $\mathrm{G}$, denoted by $\mathrm{Kf}(\mathrm{G})[1]$, is the sum of resistance distance between all pairs of vertices in G. The original index based on distance in a graph $G$ is the famous Wiener index W(G) [2], which counts the sum of distance between all pairs of vertices in G. Klein and Randic proved that $\mathrm{Kf}(\mathrm{G}) \leq$ $\mathrm{W}(\mathrm{G})$ with equality if and only if $\mathrm{G}$ is a tree. Kirchhoff index is a structure-descriptor based on resistance distance. The investigation on the Kirchhoff index of graph is an important topic in the theory of graph. It is difficult to implement some algorithms to compute resistant distance and Kirchhoff index in a graph from their computational complexity. Hence, it makes sense to find closed-form formulae for the Kirchhoff index[1,3]. In present, to compute resistance distances, various methods have been developed, and relevant formulae for resistance distance have been given for some classes of graphs, and some relevant indices to Kirchhoff index are also discussed [3-11].

The bicycle graphs are connected graphs whose number of vertices is one more than the number of edges. The first and second classes of these graphs have been discussed, and their ordering relations and extreme graphs are obtained [12]. In this paper, we will pay attention to the third class of graphs, i.e. the theta shape graphs(as shown in Fig.1 (1)). We denote the theta shape graph with n vertices by $\Theta_{n}$. $\Theta_{n}^{t}$ denotes the theta shape graph with $\mathrm{n}$ vertices, and the number of the vertices on the essential circles is $\mathrm{t}$. For the theta shape graphs, the ordering rations of their Kirchhoff indices remain open. By the graph transformations, we find some ordering relations of Kirchhoff indices and discuss the minimal Kirchhoff index for this class of graphs.

\section{Main Results}

\subsection{Three graph transformations and some ordering relations}

\footnotetext{
+ Corresponding author. Tel.: +09716307622; fax: +09716307622.

E-mail address: haizhenr@126.com
} 
Let $G$ be a simple undirected graph with the vertex set $V(G)$. We denote the number of the vertices of $G$ by $|\mathrm{V}(\mathrm{G})|$, and we use $\mathrm{u} \in \mathrm{V}(\mathrm{G})$ and $\mathrm{V}(\mathrm{G}) \backslash \mathrm{u}$ respectively to denote a vertex $\mathrm{u}$ of $\mathrm{G}$ and a vertex set that arises from $\mathrm{G}$ by deleting the vertex $\mathrm{u}$. Denote $\mathrm{d}(\mathrm{u}, \mathrm{v})$ and $\mathrm{r}(\mathrm{u}, \mathrm{v})($ or $\Omega(u, v)$ in an electrical network $\mathrm{N})$, respectively, by the distance and the resistance distance between two vertices $\mathrm{u}, \mathrm{v}$ of $\mathrm{G}$. The sum of the resistance distance from $\mathrm{v}_{\mathrm{i}}$ to other vertices of $\mathrm{G}$ is denoted by $K f_{v_{i}}(G)$. The following lemmas will be used in sequel:

Lemma 1.1([13]) Let $T$ be a tree with $n$ vertices different from the path with $n$ vertices denoted by $P_{n}$ and the star with $n$ vertices denoted by $\mathrm{S}_{\mathrm{n}}$. Then $\mathrm{W}\left(\mathrm{S}_{\mathrm{n}}\right)<\mathrm{W}(\mathrm{T})<\mathrm{W}\left(\mathrm{P}_{\mathrm{n}}\right)$.

Lemma 1.2([12]) Let $\mathrm{x}$ be a cut vertex of a connected graph $\mathrm{G}$ such that $\mathrm{G}-\mathrm{x}$ has exactly two branches $\mathrm{G}_{1}$ and $\mathrm{G}_{2}$. Let $G_{i}^{\prime}$ be the subgraph induced by $G_{i} \cup\{x\}(i=1,2)$. Then

$$
K f(G)=K f\left(G_{1}^{\prime}\right)+K f\left(G_{2}^{\prime}\right)+\left(\left|V\left(G_{1}^{\prime}\right)\right|-1\right) K f_{x}\left(G_{2}^{\prime}\right)+\left(\left|V\left(G_{2}^{\prime}\right)\right|-1\right) K f_{x}\left(G_{1}^{\prime}\right)
$$

Lemma 1.3([13]) The Kirchhoff Index of a tree is equivalent to its Wiener index.

Lemma 1.4([14,15]) For a circle $G, K f(G)=\left(n^{3}-n\right) / 12$.

Lemma 1.5([11]) Let $G$ be a connected graph with $e=i j$ being an edge. Let $G^{\prime}=G-e$ and $G^{*}=G l e$. Then

$$
\Omega(p, q)=[1-\Omega(i, j)] \cdot \Omega^{\prime}(p, q)+\Omega(i, j) \Omega^{*}(p, q)
$$

Lemma 1.6([11]) Let $\Omega$ and $\Omega^{\prime}$ be resistance distance functions for edge-weighted connected graphs $\mathrm{G}$ and $\mathrm{G}^{\prime}$ which are the same except for the weights $\omega$ and $\omega^{\prime}$ on an edge e=ij. Then

$$
K f\left(G^{\prime}\right)=K f(G)-\frac{\delta n \sum_{k=1}^{n}[\Omega(i, k)-\Omega(j, k)]^{2}-\delta\left[\sum_{k=1}^{n} \Omega(i, k)-\sum_{k=1}^{n} \Omega(j, k)\right]^{2}}{4[1+\delta \Omega(i, j)]}
$$

where $\delta=\omega^{\prime}-\omega$.

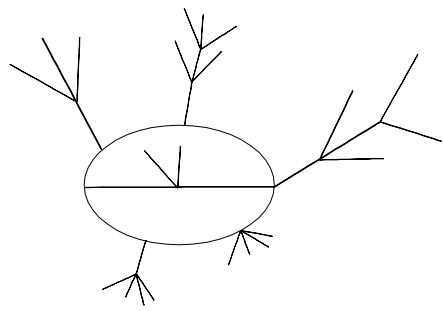

(1)

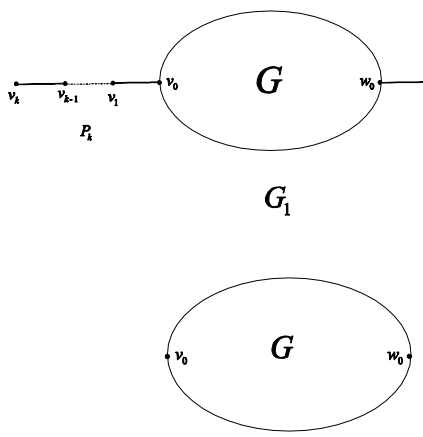

(4)

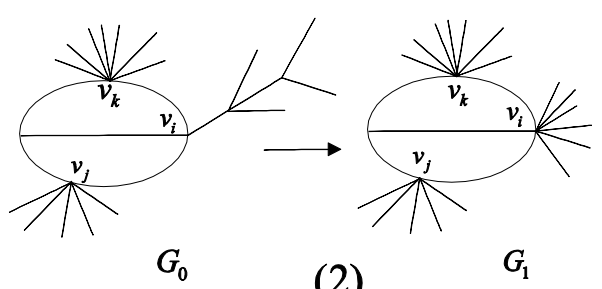

(2)

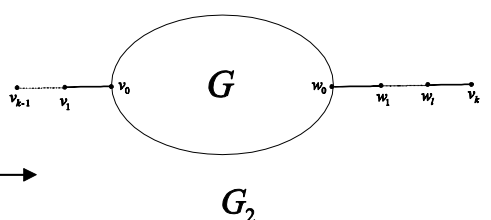

(3)

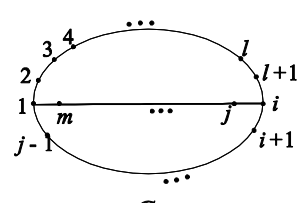

$G$

(5)

Fig. 1 The theta shape graph and the transformations I-II

- Claim 1. $\mathrm{G}_{0} \in \Theta_{n}$ is the graph with the minimal Kirchhoff index, for any cut vertex $v_{i}$ with hanging tree $T_{i}$ in $G_{0}, T_{i}$ is a star, and $v_{i}$ is its central vertex.

Proof. Assuming that $T_{i}$ is not a star in $G_{0}$ (See Fig.1 (2)), then we apply the following graph transformation I:

Transformation I: We obtain $G_{1}$ from $G_{0}$ by deleting all the edges of $T_{i}$ in $G_{0}$ and joining them with $v_{i}$, that is to say, we change $T_{i}$ in $G_{0}$ to the star $S_{i}$ attaching to $v_{i}$ in $G_{1}$.

By Lemma 1.1, we have $\mathrm{Kf}\left(\mathrm{S}_{\mathrm{i}}\right)<\mathrm{Kf}\left(\mathrm{T}_{\mathrm{i}}\right)$ and $K f_{v_{i}}\left(S_{i}\right)<K f_{v_{i}}\left(T_{i}\right)$. $\mathrm{v}_{\mathrm{i}}$ is a cut vertex of $\mathrm{G}_{0}$. Assume that $\mathrm{G}_{01}$ and $\mathrm{T}_{\mathrm{i}}$ are two branches of $\mathrm{G}_{0}-\mathrm{V}_{\mathrm{i}} . G_{01}^{\prime}$ and $T_{i}^{\prime}$ are the subgraphs induced by $V\left(G_{01}\right) \bigcup\left\{v_{i}\right\}$ and $V\left(T_{i}\right) \bigcup\left\{v_{i}\right\}$, respectively. By Lemma 1.2, we have 


$$
K f\left(G_{0}\right)=K f\left(G_{01}^{\prime}\right)+K f\left(T_{i}^{\prime}\right)+\left(\left|V\left(G_{01}^{\prime}\right)\right|-1\right) K f_{v_{i}}\left(T_{i}^{\prime}\right)+\left(\left|V\left(T_{i}^{\prime}\right)\right|-1\right) K f_{v_{i}}\left(G_{01}^{\prime}\right) .
$$

We denote that $\mathrm{G}_{11}$ and $\mathrm{S}_{\mathrm{i}}$ are two branches of $G_{1}-v_{i}, G_{11}^{\prime}$ and $S_{i}^{\prime}$ are the subgraphs induced by $V\left(G_{11}\right) \bigcup\left\{v_{i}\right\}$ and $V\left(S_{i}\right) \bigcup\left\{v_{i}\right\}$, respectively. Also by Lemma 1.2,

$$
K f\left(G_{1}\right)=K f\left(G_{11}^{\prime}\right)+K f\left(S_{i}^{\prime}\right)+\left(\left|V\left(G_{11}^{\prime}\right)\right|-1\right) K f_{v_{i}}\left(S_{i}^{\prime}\right)+\left(\left|V\left(S_{i}^{\prime}\right)\right|-1\right) K f_{v_{i}}\left(G_{11}^{\prime}\right) .
$$

Since $K f\left(G_{01}^{\prime}\right)=K f\left(G_{11}^{\prime}\right),\left|V\left(G_{01}^{\prime}\right)\right|=\left|V\left(G_{11}^{\prime}\right)\right|$ and $\left|V\left(T_{i}\right)\right|=\left|V\left(S_{i}\right)\right|$. Then we get $\mathrm{Kf}\left(\mathrm{G}_{1}\right)<\mathrm{Kf}\left(\mathrm{G}_{0}\right)$. It is a contradiction.

- Claim 2. For $k \leq l$, the transformation II is shown as in Fig.1 (3), then $K f\left(G_{2}\right)>K f\left(G_{1}\right)$.

Proof. By Lemma 1.3, we have

$$
\begin{aligned}
K f_{v_{k}}\left(G_{1}\right) & =\sum_{u \in V\left(P_{k}\right)} r\left(v_{k}, u\right)+\sum_{u \in V(G)} r\left(v_{k}, u\right)+\sum_{u \in V\left(P_{l}\right)} r\left(v_{k}, u\right)=\frac{k(k-1)}{2}+\sum_{u \in V(G)}\left[k+r\left(v_{0}, u\right)\right]+\sum_{i=1}^{l}\left[k+r\left(v_{0}, w_{0}\right)+i\right] \\
& =\frac{k(k-1)}{2}+k|V(G)|+K f_{v_{0}}(G)+k l+\frac{l(l+1)}{2}+l \cdot r\left(v_{0}, w_{0}\right)
\end{aligned}
$$

and

$$
\begin{aligned}
K f_{w_{l}}\left(G_{1}\right) & =\sum_{u \in V\left(P_{k}\right)} r\left(w_{l}, u\right)+\sum_{u \in V(G)} r\left(w_{l}, u\right)+\sum_{u \in V\left(P_{l}\right)} r\left(w_{l}, u\right)=\frac{l(l-1)}{2}+\sum_{u \in V(G)}\left[l+r\left(w_{0}, u\right)\right]+\sum_{i=1}^{k}\left[l+r\left(w_{0}, v_{0}\right)+i\right] \\
& =\frac{l(l-1)}{2}+l|V(G)|+K f_{w_{0}}(G)+k l+\frac{k(k+1)}{2}+k \cdot r\left(w_{0}, v_{0}\right)
\end{aligned}
$$

So, $K f_{w_{i}}\left(G_{1}\right)-K f_{v_{k}}\left(G_{1}\right)=(l-k)\left[|V(G)|-r\left(v_{0}, w_{0}\right)-1\right]$. Since $|V(G)|-1 \geq r\left(v_{0}, w_{0}\right)$ and $l-k \geq 0$.

Then $K f_{v_{k}}\left(G_{1}\right) \leq K f_{w_{l}}\left(G_{1}\right)$. Thus, $K f_{v_{k}}\left(G_{2}\right)=K f_{w_{i}}\left(G_{1}\right)-r_{G_{1}}\left(w_{l}, v_{k}\right)+\left|V\left(G_{1}\right)\right|-1>K f_{w_{l}}\left(G_{1}\right)$. Therefore $K f_{v_{k}}\left(G_{2}\right)>K f_{v_{k}}\left(G_{1}\right)$.

Since $K f\left(G_{2}\right)-K f\left(G_{1}\right)=K f_{v_{k}}\left(G_{2}\right)-K f_{v_{k}}\left(G_{1}\right)$. Then we get $K f\left(G_{2}\right)>K f\left(G_{1}\right)$.

- Claim 3. $\mathrm{G}_{0} \in \Theta_{n}$ is the graph with the minimal Kirchhoff index, the central vertices of the stars must have three neighbors besides the leaves of the stars.

Proof. In Fig.1 (4), for the graph $G$ there is an automorphism $\Phi$ such that $\Phi\left(\omega_{0}\right)=\Phi\left(v_{0}\right),|V(G)| \geq 3$, and $d\left(v_{0}, w_{0}\right) \geq 2$. Then, for any $\mathrm{u} \in \mathrm{V}(\mathrm{G}), r\left(v_{0}, u\right)=r\left(\omega_{0}, u\right)$. In Fig.1 (5), note that $i<j \leq m$, for any vertex $\mathrm{q} \in$ $\mathrm{V}(\mathrm{G})$, by Lemma 1.5 we have $r_{G}(l, q)=\left[1-r_{G}(l, l+1)\right] \cdot r_{G_{1}}(l, q)+r_{G}(l, l+1) \cdot r_{G_{2}}(l, q)$.

If $1 \leq l \leq[i / 2]$ then $r_{G_{1}}(l, q)<r_{G_{1}}(l+1, q)$; and if $[i / 2]<l<i$ then $r_{G_{1}}(l, q)>r_{G_{1}}(l+1, q)$. So the relation of $K f_{v_{l}}(G)$ and $K f_{v_{t}+1}(G)$ is $K f_{v_{i}}(G)<K f_{v_{i}+1}(G)$ when $1 \leq l \leq[i / 2]$, and $K f_{v_{l}}(G)>K f_{v_{t}+1}(G)$ when $[i / 2]<l<i$. Thus, we get the graph $\mathrm{G}_{0}$ as shown in Fig.1 (6).

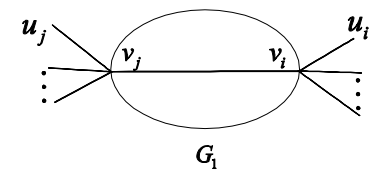

(7)

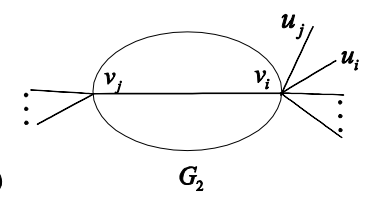

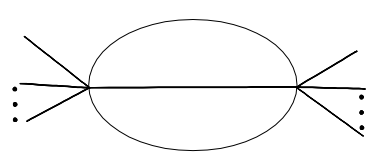

(6)

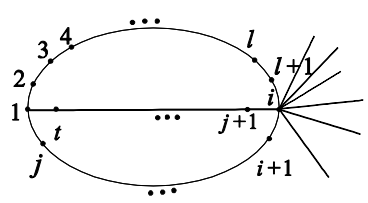

(8)

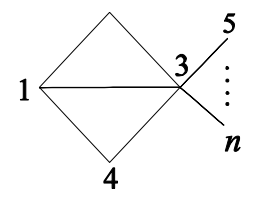

(9)

Fig. 2 The transformation III and the extreme graph

- Claim 4. In Fig.2 (7), we assume that $T_{i}$ and $T_{j}$ are nontrivial stars in $G_{1}$, which are hanging to the central vertices $v_{i}$ and $v_{j}$, respectively, and assume that $u_{i}$ is a leave of $T_{i}$ and $u_{j}$ is a leave of $T_{j}$. When $K f_{u_{i}}\left(G_{1}\right) \leq K f_{u_{j}}\left(G_{1}\right)$. We apply the transformation III to $\mathrm{G}_{1}$. The resulting graph is $\mathrm{G}_{2}$ (as shown in Fig. 2 (7)). Then $K f\left(G_{2}\right)<K f\left(G_{1}\right)$.

Proof. For any two vertices $v_{k}, v_{l} \in V\left(G_{t}\right) \backslash u_{j}(\mathrm{t}=1,2)$, we know that $r_{G_{1}}\left(v_{k}, v_{l}\right)=r_{G_{2}}\left(v_{k}, v_{l}\right)$ and

$$
K f_{u_{j}}\left(G_{2}\right)=K f_{u_{i}}\left(G_{2}\right)=K f_{u_{i}}\left(G_{1}\right)-r_{G_{1}}\left(u_{i}, u_{j}\right)+2<K f_{u_{i}}\left(G_{1}\right) \leq K f_{u_{j}}\left(G_{1}\right) .
$$

So, $K f\left(G_{2}\right)=K f\left(G_{1}\right)-K f_{u_{j}}\left(G_{1}\right)+K f_{u_{j}}\left(G_{2}\right)<K f\left(G_{1}\right)$.

By Claim 4, we have the following Claim 5: 
- Claim 5. $\mathrm{G}_{0} \in \Theta_{n}$ is the graph with the minimal Kirchhoff index, $\mathrm{S}_{\mathrm{i}}$ is the only nontrivial star with the central vertex $v_{i}$.

Claims 1, 3 and 5 demonstrate the following Theorem .

Theorem If $G \in \Theta_{n}$, and $G \neq \Theta_{n}^{t}\left(\Theta_{n}^{t}\right.$ is shown as in Fig.2 (8), the number of the vertices on the essential circles is $\mathrm{t})$, then $K f(G)>K f\left(\Theta_{n}^{t}\right)$.

\subsection{The minimal Kirchhoff index and the corresponding graph}

We consider the graph $\Theta_{n}^{t}$. For $\Theta_{n}^{t}$, if $\mathrm{t}=4, \mathrm{i}=3$ and $\mathrm{j}=4$, denote $\Theta_{n}^{t}$ by $G_{t_{1}}$; and if $\mathrm{t}=5, \mathrm{i}=3$ and $\mathrm{j}=5$, denote $\Theta_{n}^{t}$ by $G_{i_{2}}$; and if $\mathrm{t}=6, \mathrm{i}=4$ and $\mathrm{j}=6$, denote $\Theta_{n}^{t}$ by $G_{t_{3}}$; and if $\mathrm{t}=6, \mathrm{i}=3$ and $\mathrm{j}=6$, denote $\Theta_{n}^{t}$ by $G_{i_{4}}$. According to that Lemma 1.6, we obtain that $K f\left(G_{i,}\right)=15 n / 4-11$ by choosing the edge $e_{13}, K f\left(G_{i,}\right)=53 n / 11-175 / 11$ by choosing the edge $e_{13}, K f\left(G_{t_{1}}\right)=89 n / 15-107 / 5$ by choosing the edge $e_{14}$, and $K f\left(G_{t_{4}}\right)=87 n / 14-317 / 14$ by choosing the edge $e_{13}$. So, by simple comparing we find that $K f\left(G_{i_{1}}\right)<K f\left(G_{i_{2}}\right)(\mathrm{n} \geq 4.60), \quad K f\left(G_{i_{2}}\right)<K f\left(G_{i_{1}}\right)(\mathrm{n} \geq 4.93)$ and $K f\left(G_{t_{3}}\right)<K f\left(G_{t_{i}}\right)(\mathrm{n} \geq 4.43)$. Thus, when $\mathrm{n} \geq 5$, we have

$$
K f\left(G_{i_{1}}\right)<K f\left(G_{i_{2}}\right)<K f\left(G_{i_{3}}\right)<K f\left(G_{i_{4}}\right) .
$$

In general, if the shortest length of three internally paths in $\Theta_{n}^{t}$ is one, we denote this shortest path by $e_{13}$, then by Lemma 1.6 we get

$$
K f\left(G^{\prime}\right)=K f(G)-\frac{n \sum_{k=1}^{n}[\Omega(i, k)-\Omega(j, k)]^{2}-\left[\sum_{k=1}^{n} \Omega(i, k)-\sum_{k=1}^{n} \Omega(j, k)\right]^{2}}{4[1+\Omega(i, j)]}
$$

and $\Omega(1,3)=2(t-2) / t$. And by Lemma 1.5 we have $K f(G)=\left(t^{3}-t\right) / 12+(n-t)\left(t^{2}+11\right) / 6$. Also, if $\mathrm{k}=1$ then $[\Omega(1,1)-\Omega(3,1)]^{2}=4(t-2)^{2} / t^{2}$; and if $\mathrm{k}=2$ then $[\Omega(1,2)-\Omega(3,2)]^{2}=0$; and if $\mathrm{k}=3$ then $[\Omega(1,3)-\Omega(3,3)]^{2}=4(t-2)^{2} / t^{2}$; and if $4 \leq \mathrm{k} \leq \mathrm{t}$ then $[\Omega(1, k)-\Omega(3, k)]^{2}=[(k-1)(t-k+1) / t-(k-3)(t-k+3) / t]^{2}=(2 t-4 k+8)^{2} / t^{2} ;$ and if $\mathrm{t}+1 \leq \mathrm{k} \leq \mathrm{n}$ then

$$
\sum_{k=t+1}^{n}[\Omega(1, k)-\Omega(3, k)]^{2}=(n-t)[\Omega(1,3)]^{2}=4(n-t)(t-2)^{2} / t^{2}
$$

Since $\left[\sum_{k=1}^{n} \Omega(1, k)-\sum_{k=1}^{n} \Omega(3, k)\right]^{2}=\frac{4(n-t)^{2}(t-2)^{2}}{t^{2}}$. Thus,

$$
K f\left(G^{\prime}\right)=\frac{-3 t^{4}+(6 n+16) t^{3}-(24 n+117) t^{2}+(126 n+140) t-144 n}{12(3 t-4)},
$$

and the derivative of $K f\left(G^{\prime}\right)$ about $\mathrm{t}$ is

$$
\frac{-27 t^{4}+144 t^{3}-543 t^{2}+936 t-560+n\left(36 t^{3}-144 t^{2}+192 t-72\right)}{12(3 t-4)^{2}}
$$

We note that the denominator of above fraction is a positive number, and when $t \geq 4$ we have $36 t^{3}-144 t^{2}+192 t-72=12 t\left(3 t^{2}-12 t+16\right)-72>0$. Since $\mathrm{t} \leq \mathrm{n}$. Then

$$
\begin{aligned}
& -27 t^{4}+144 t^{3}-543 t^{2}+936 t-560+n\left(36 t^{3}-144 t^{2}+192 t-72\right) \\
\geq & -27 t^{4}+144 t^{3}-543 t^{2}+936 t-560+t\left(36 t^{3}-144 t^{2}+192 t-72\right) \\
= & 9 t^{2}\left(t^{2}-39\right)+864 t-560
\end{aligned}
$$

If $\mathrm{t} \geq 5$ then $9 t^{2}\left(t^{2}-39\right)+864 t-560>0$, thus $K f\left(G^{\prime}\right)$ is increasing with the increase of $\mathrm{t}$. If $\mathrm{t}=4$ then $9 t^{2}\left(t^{2}-39\right)+864 t-560<0$, by comparing $G_{t_{1}}$ and $G_{i_{2}}$ we know that $K f\left(G_{i_{2}}\right)>K f\left(G_{t_{1}}\right)$. Therefore, if the shortest length of three internally paths in $\Theta_{n}^{t}$ is one, then the minimal Kirchhoff index is $15 n / 4-11$ and the corresponding graph is $\Theta_{n, n-4}$ (as shown in Fig.2 (9)).

\section{Acknowledgements}

This work is partially supported by the National Natural Science Foundation of China (Grant Nos. 11551003) and the Qinghai Natural Science Foundation of China (Grant Nos. 2015-ZJ-911)

\section{References}

[1] D. J. Klein, M. Randic, Resistance distance, J. Math. Chem. 1993, 12: 81-95.

[2] H. Wiener, Structural determination of paraffin boiling points, J. Amer. Chem. Soc. 1947, 69: 17-20. 
[3] Y. J. Yang and D. J. Klein, A recursion formula for resistance distance and its applications, Discrete Appl. Math. 2013, 161: 2702-2715.

[4] S. B. Chen, Q. Chen, X. Cai and Z. J. Guo, Maximal Degree Resistance Distance of Unicycle Graphs, MATCH Commun. Math. Comput. Chem. 2016, 75: 157-168.

[5] S. B. Huang, J. Zhou, C. J. Bu, Some Results on Kirchhoff Index and Degree-Kirchhoff Index, MATCH Commun. Math. Comput. Chem. 2016, 75: 207-222.

[6] M. Bianchi, A. Cornaro, J. L. Palacios, J. M. Renom and A. Torriero, Revisiting Bounds for the Multiplicative Degree-Kirchhoff Index, MATCH Commun. Math. Comput. Chem. 2016, 75: 227-231.

[7] H. P. Zhang and Y. J. Yang, Resistance distance and Kirchhoff index in circulate graphs, Int. J. Quantum Chem. 2007, 107: 330-339.

[8] H. P. Zhang, Y. J. Yang and C. W. Li, Kirchhoff index of composite graphs, Discrete Appl. Math. 2009, 157: 2918-2927.

[9] R. B. Bapat and S. Gupta, Resistance distance on wheels and fans, Indian J. Pure Appl. Math. 2010, 41: 1-13.

[10] X. Gao, Y. Luo and W. Liu, Resistance distances and the Kirchhoff index in Cayley graphs, Discrete Appl. Math. 2011,159: 2050-2057.

[11] H. P. Zhang, Y. J. Yang, Kirchhoff Index of Linear Hexagonal Chains, International Journal of Quantum Chemistry, 2008, 108: 503-512.

[12] X. Y. Jiang, The Kirchhoff Index extreme of the bicycle graphs, The master's degree thesis, 2008.

[13] R. C. Entringer, D. E. Jackson and D. A. Snyder, Distance in graphs, Czechoslovak Math. J. 1976, 26: $283-296$.

[14] I. Lukovits, S. Nikolic, N. Trinajstic, Resistance distance in regular graphs, Int. J. Quantum Chem. 1999, 71: 217 225.

[15] D. J. Klein, Lukovits and I. Gutman, On the definition of the hyper-wiener index for cycle containing structures, $J$. Chem. Inf. Comput. Sci. 1995, 35: 50-52. 